\title{
Subliminally Presented and Stored Objects Capture Spatial Attention
}

\author{
Duncan E. Astle, Anna C. Nobre, and Gaia Scerif \\ Department of Experimental Psychology, University of Oxford, Oxford, OX1 3UD, United Kingdom
}

When objects disappear from view, we can still bring them to mind, at least for brief periods of time, because we can represent those objects in visual short-term memory (VSTM) (Sperling, 1960; Cowan, 2001). A defining characteristic of this representation is that it is topographic, that is, it preserves a spatial organization based on the original visual percept (Vogel and Machizawa, 2004; Astle et al., 2009; Kuo et al., 2009). Recent research has also shown that features or locations of visual items that match those being maintained in conscious VSTM automatically capture our attention (Awh and Jonides, 2001; Olivers et al., 2006; Soto et al., 2008). But do objects leave some trace that can guide spatial attention, even without participants intentionally remembering them? Furthermore, could subliminally presented objects leave a topographically arranged representation that can capture attention? We presented objects either supraliminally or subliminally and then 1 s later re-presented one of those objects in a new location, as a "probe" shape. As participants made an arbitrary perceptual judgment on the probe shape, their covert spatial attention was drawn to the original location of that shape, regardless of whether its initial presentation had been supraliminal or subliminal. We demonstrate this with neural and behavioral measures of memory-driven attentional capture. These findings reveal the existence of a topographically arranged store of "visual" objects, the content of which is beyond our explicit awareness but which nonetheless guides spatial attention.

\section{Introduction}

Typically, visual short-term memory (VSTM) tasks require participants to search voluntarily their stored representations. Their task is usually to decide whether a probe item was part of a preceding memory array (Fabiani et al., 2003; Vogel and Machizawa, 2004; Astle et al., 2009; Kuo et al., 2009). VSTM has been shown to retain a topographic organization (Fabiani et al., 2003; Astle et al., 2009; Kuo et al., 2009; Luria et al., 2010). Recent experiments have shown that the voluntary selection of an item from memory leads to event-related potentials (ERPs) that are relatively more negative over posterior scalp contralateral versus ipsilateral to the original object location between 200 and 300 ms (Fabiani et al., 2003; Astle et al., 2009; Eimer and Kiss, 2009; Kuo et al., 2009; Dell'Acqua et al., 2010). The effect resembles the lateralized ERP differences obtained when participants search for objects in perceptual input, known as the N2pc (Luck and Hillyard, 1994; Woodman and Luck, 1999), which is thought to reflect spatially specific biases on visual cortices (Hopf et al., 2004) after target selection processes in areas such as the posterior parietal cortex (Fuggetta et al., 2006) and frontal eye fields (Cohen et al., 2009).

In addition to being voluntarily deployed while actively searching memory, attention can be captured involuntarily by the contents of VSTM (Awh and Jonides, 2001; Olivers et al.,

Received Nov. 17, 2009; revised Jan. 14, 2010; accepted Jan. 24, 2010.

D.E.A. is supported by a Fellowship from the Economic and Social Research Council. D.E.A. and G.S. are supported by a project grant from the John Fell Fund (Oxford University Press). G.S. and A.C.N. are supported by project grants from the Wellcome Trust.

Correspondence should be addressed to Duncan E. Astle, Department of Experimental Psychology, University of 0xford, South Parks Road, 0xford, 0X1 3UD, UK. E-mail: duncan.astle@psy.ox.ac.uk.

DOI:10.1523/JNEUROSCI.5701-09.2010

Copyright $\odot 2010$ the authors $\quad 0270-6474 / 10 / 303567-05 \$ 15.00 / 0$
2006; Soto et al., 2008). We therefore asked whether, just as voluntary selection from VSTM recruits spatial-biasing neural mechanisms, the involuntary capture of attention by items stored in VSTM might also elicit an N2pc. We also explored whether subliminally presented objects leave a topographically organized trace that cannot be accessed consciously but that can nonetheless capture our attention. Across a series of experiments, we demonstrate that indeed this is the case. To test whether the contents of a past subliminal display could exert a spatial bias on attention, we presented participants with a supraliminal or subliminal "memory" array of line shapes and, after $1 \mathrm{~s}$, presented them with a test probe. On a proportion of trials, the test probe was one of the original memory array shapes, presented earlier that trial. Regardless of whether the memory array had been presented subliminally or supraliminally, when one of the shapes in the array reappeared as the test probe, participants' attention was drawn back to the original location of that shape. This was evidenced by a clear neural marker of spatial biasing, the N2pc: a greater negativity contralateral to the original location of that shape in both subliminal and supraliminal arrays (experiment 1). We also observed the direct behavioral consequences of this memory-driven attentional bias using a dot-probe experiment (experiment 3). Despite this effect on spatial attention, during the subliminal condition, participants were unable to recognize consciously the test probe as being one of the memory-array shapes (experiment 1) and showed no conscious bias as to the most likely location of the test probe in the previous array (experiment 2).

\section{Materials and Methods}

All the experiments described had the same basic trial structure. We presented a memory array of two items either subliminally $(63 \mathrm{~ms})$ or supraliminally ( $243 \mathrm{~ms}$ ), sandwiched between two pattern masks com- 
prising visual noise (70 ms) (see Fig. 1 A). After a delay of $800-1200 \mathrm{~ms}$, a test probe shape was presented. Participants had to perform a judgment on this test probe shape.

All shapes were constructed from straight lines, subtended $2^{\circ} \times 2^{\circ}$ of visual angle at their highest/widest point, and were positioned along the outer edge of a diagonal (upper left and lower right or upper right and lower left) of an invisible $2 \times 2$ matrix that subtended $\sim 2.6^{\circ} \times 2.4^{\circ}$. The pattern masks were constructed by randomly overlaying 15 of these straight lines, each of $2^{\circ}$ in length, over one another. Across all trials, each array contained two shapes that were either symmetrical or asymmetrical along the vertical midline, with shapes being drawn from a set of 12 . These stimuli were used for all experiments.

Experiment 1: neural consequences of memory-driven attentional capture on a perceptual judgment task. The electrophysiological recording session started and concluded with an explicit-memory search task. This was designed to assess the liminality of the memory-array shapes. Participants' ( $n=13$; mean age of 25.1 years; six males) task was to decide whether the test probe had been present in the original memory array. On half of the trials, the test probe appeared in the memory array. They responded with their right hand, with their index finger corresponding to "present" and their middle finger corresponding to "absent." $d$ ' indexed participants' sensitivity to the presence of the test probe in the preceding memory array.

During an electrophysiological recording session, participants performed 40 blocks of 10 trials. The trial sequence was identical to that used in the explicit-memory search task. This time, participants' task was to judge the symmetry along the vertical midline of the test probe; this meant that the task was unrelated to the contents of the memory array. On two-thirds of trials, the test probe was one of the shapes in the preceding memory array. These trials contributed to the ERP analyses.

The electroencephalogram was recorded continuously $(1000 \mathrm{~Hz}$ analog-to-digital rate; $0.1-300 \mathrm{~Hz}$ bandwidth; right-mastoid reference), subsequently re-referenced to the algebraic average of the right and left mastoids, and low-pass filtered at $40 \mathrm{~Hz}$. Trials contaminated by blinks or eye movements were removed. Because the task performed on the probe was entirely irrelevant to the characteristics of the memory array, we did not discard behavioral errors from the ERP. That is, the purpose of the ERP comparison was not to explore the mechanisms by which participants distinguish symmetrical and asymmetrical shapes but rather to test whether processing of the test probe was influenced, in a spatially specific way, by having "seen" that item before. We compared waveforms locked to the onset of the probe item over posterior contralateral versus ipsilateral electrode locations relative to the position of the item in the memory array that matched the probe. We submitted the mean voltage across the 230-280 ms window to a repeated-measures ANOVA for factors supraliminal versus subliminal, contralateral versus ipsilateral hemisphere relative to the item location in the memory array, and electrode (three levels, PO7/8, P7/8, and O1/2). The time window was selected on the basis of previous work examining the N2pc effect (Astle et al., 2009). All statistical tests were corrected for the potential nonsphericity of EEG data.

For one-third of trials, the memory array did not contain the test probe. These trials were used for an additional test of liminality: for half of these probe-absent trials, the symmetry of the probe shape was congruent with the symmetry of the array items, i.e., the probe and array shapes were all either symmetrical or asymmetrical. For the other half of the probe-absent trials, the symmetry of the probe shape was incongruent with the symmetry of the memory array items. Were participants able to see the memory array shapes, we might expect their symmetry to prime participants' response to the test probe, i.e., we might see a difference between congruent and incongruent trials.

Experiment 2: location decision task. Although participants might be unable to recognize consciously the test probe per se, they might nonetheless have had some conscious bias toward which memory array shape was most likely to have been the test probe. This bias might not be measured by our previous tests of liminality used in experiment 1 but might nonetheless drive the electrophysiological effects observed. To check whether this was the case, 11 of the participants who took part in experiment 1 also performed a separate experiment in a subsequent ses- sion. The trial sequence was identical to experiment 1 . On each trial, the test probe was always one of the two memory array shapes, and participants' task was to decide which of the two memory array shapes was most likely to match the test probe. Participants responded with their lefthand and right-hand index fingers, depending on whether they thought the test probe was most likely to have been the left-hand or right-hand memory-array shape. We used $d^{\prime}$ to establish how sensitive, relative to chance, participants were at selecting the appropriate location of the test probe in the preceding memory array. We reasoned that this would give us the best chance of identifying whether participants had any conscious bias for which shape was most likely to be the test probe, even if they could not consciously recognize the test probe shape.

Experiment 3: behavioral effects of memory-driven attentional capture. There is a limitation to the electrophysiological experiment (experiment 1) deserving consideration: although lateralized electrophysiological biases have been reliably reproduced when participants engage in attentional selection (Luck and Hillyard, 1994; Woodman and Luck, 1999), it does not logically follow that, if we observe them, attentional selection has been engaged. We therefore conducted a follow-up behavioral experiment to test the spatiotopic allocation of attention directly. Thirteen participants (eight had also participated in experiments 1 and 2) performed a follow-up dot-probe experiment. Trials (300 organized into blocks of 10) were identical to those in the previous experiment, except for the addition of the dot probe, presented for $90 \mathrm{~ms}$ either 150 or 400 $\mathrm{ms}$ after the onset of the test-probe shape. Participants' task was to respond as to whether a small section was missing from the right or left side of the dot, with their right-hand index and middle fingers, respectively. The memory array and test probe shapes were essentially irrelevant to the task the participants were performing. On each trial, one of the memoryarray shapes would appear as the test probe, and the dot was as likely to appear in the original location of these shapes as in the location previously occupied by the other array shape. If, when the test probe appears, participants' attention is allocated to the original location of the probematching item in the preceding subliminal memory array, then there should be a behavioral facilitation for subsequent stimuli appearing in this "attended" location relative to other locations (Fukuda and Vogel, 2009).

\section{Results}

\section{Experiment 1: neural consequences of supraliminal and subliminal memory-driven attentional capture on a perceptual judgment}

Explicit-memory search task

This was conducted before and after the electrophysiological recording session to ensure that the contents of the arrays were not consciously detectable in the subliminal condition. Participants judged whether the test probe had been present in the memory array. When the memory array was presented for only $63 \mathrm{~ms}$, participants' performance remained at chance $\left(d^{\prime}=-0.13, t_{(12)}=\right.$ $0.895, p=0.388)$. In contrast, when the memory array was presented for $243 \mathrm{~ms}$, participants' memory-based discrimination was significantly better than chance $\left(d^{\prime}=1.91, t_{(12)}=5.835, p<\right.$ 0.001) (Fig. 1C).

Participants had not developed the ability to see the memory arrays during the lengthy recording session: at the end, just as at the start of the recording session, participants were unable to perceive the contents of the arrays presented for $63 \mathrm{~ms}$ above a chance level $\left(d^{\prime}=-0.31, t_{(12)}=-1.296, p=0.219\right)$ and were able to do so when the arrays were presented for $243 \mathrm{~ms}\left(d^{\prime}=\right.$ 2.00, $t_{(12)}=7.483, p<0.001$ ) (Fig. 1C).

\section{Electrophysiological markers}

Having validated the manipulation of liminality, we proceeded to recording electrical brain activity from the same participants, using scalp electrodes, while they viewed the same subliminal and supraliminal stimuli (Fig. $1 A$ ). Although the memory array had been completely irrelevant to task performance, when the test 


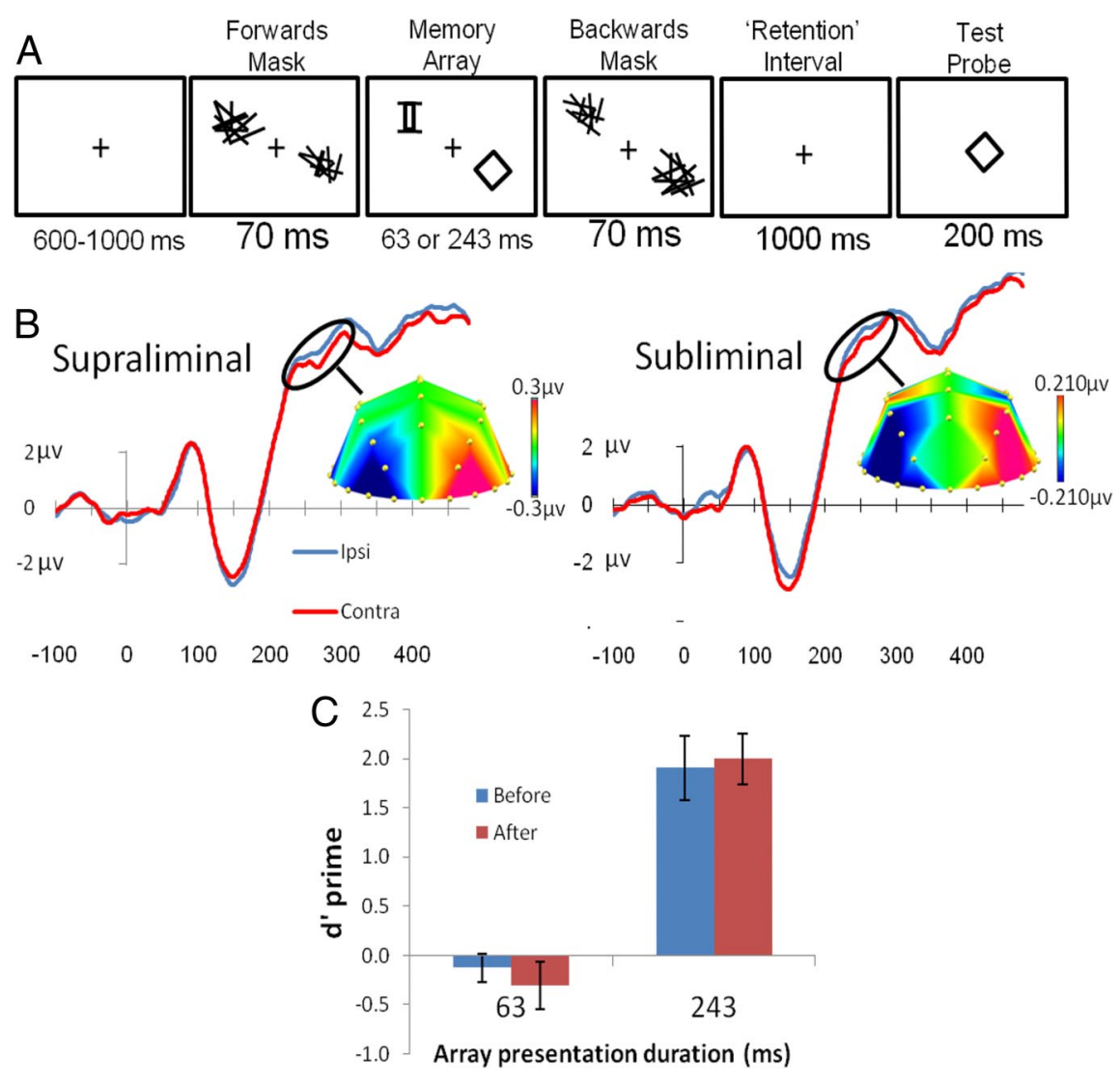

Figure 1. $\quad \boldsymbol{A}$, Trial order schematic. $\boldsymbol{B}$, Grand-averaged event-related potentials comparing posterior recordings contralateral and ipsilateral to the location of the probe-matching item in the memory array, locked to the onset of the test probe. Each waveform is the average of three electrodes ( $\mathrm{P} 07 / 8, \mathrm{P} 7 / 8$, and $01 / 2)$. We found a reliable main effect of contralaterality between 230 and $280 \mathrm{~ms}$. This was also present as a simple main effect of contralaterality in both the supraliminal and subliminal conditions. There was no interaction between contralaterality and liminality or with liminality and electrode. Topographical plots show contralateral minus ipsilateral voltage in the left hemisphere; the right hemisphere shows the mirror of this. C, Participants' mean $d^{\prime}$ on the explicit-memory search task, for each duration of the memory array, before and after the electrophysiological recording session.

probe appeared, we found a reliable main effect of contralaterality between 230 and $280 \mathrm{~ms}\left(F_{(1,12)}=10.34, p=0.007\right)$. That is, we observed a negativity contralateral to the original location of the test probe item in the memory array. This effect did not interact with electrode or liminality ( $p$ values $<0.34$ ). Analyses of simple effects confirmed that, when the memory array was supraliminal, we observed the probe-locked N2pc effect $\left(F_{(1,12)}=4.70\right.$, $p=0.05$ ) (Fig. $1 B$, left). Thus, although the N2pc has been observed previously when participants search memory actively (Kuo et al., 2009), this neural marker also occurs when the contents of memory are irrelevant to the task at hand (Olivers et al., 2006). Surprisingly, we also observed the N2pc effect when the arrays were subliminal $\left(F_{(1,12)}=5.03, p=0.04\right)$ (Fig. $1 B$, right). This suggests that attention was still directed to the original location of those probe-matching objects (Luck and Hillyard, 1994; Woodman and Luck, 1999), even when participants had been unable to perceive them consciously.

Behavior during the electrophysiological session

As was outlined in Materials and Methods, we incorporated a test of liminality into the electrophysiological recording. When the memory array had been supraliminal, congruence between the symmetry of the shapes in the memory array and in the test probe facilitated behavioral performance (Fig. 2A); on probe-absent trials, participants were faster and more accurate to respond to congruent than to incongruent probes (411 and $524 \mathrm{~ms}$, respectively, $F_{(1,12)}=$ $34.07, p<0.001 ; 93$ and $62 \%$ correct, respectively, $\left.F_{(1,12)}=17.72, p=0.001\right)$. That performance was affected to such a great extent by the congruence of the memory arrays suggests that participants were attempting to process the contents of the arrays and used this information to speed response selection to the test probe, resulting in a large number of errors on incongruent trials. If participants were able to detect at least part of one shape in the subliminal condition, we would expect the same effect. However, in this case, there was no effect of whether the probe was congruent or incongruent on either reaction times (511 and $516 \mathrm{~ms}$, respectively, $\left.F_{(1,12)}=0.83, p=0.38\right)$ or error rates (90 and $92 \%$, respectively, $F_{(1,12)}=$ $0.74, p=0.41$ ) (Fig. $2 B$ ). The lack of this behavioral priming effect in the subliminal condition is strong evidence that participants could not detect the contents of the subliminal arrays during the electrophysiological recording session.

\section{Experiment 2: location decision task}

The electrophysiological effects elicited by subliminal stimuli in experiment 1 might have been driven by a conscious bias toward which memory array shape was most likely to have been the test probe. This bias might not be measured by our previous tests of liminality but might nonetheless drive the N2pc that we observed. We produced a $d^{\prime}$ measure of participants' sensitivity to the location of the test probe within the memory array. When the array was presented for $243 \mathrm{~ms}$, participants' $d^{\prime}$ scores were significantly greater than zero $\left(d^{\prime}=3.96, t_{(11)}=8.358, p<\right.$ $0.001)$. When the array was presented for $63 \mathrm{~ms}$, participants' $d^{\prime}$ scores were not significantly greater than zero $\left(d^{\prime}=0.15, t_{(11)}=\right.$ $1.591, p=0.143$ ). In addition to being unable to identify consciously the subliminal memory array shapes or to categorize their symmetry along the vertical midline, participants showed no conscious bias as to the most likely location of a particular test probe.

\section{Experiment 3: behavioral effects of memory-driven attentional capture}

Having ruled out explanations related to participants consciously perceiving the items in the subliminal arrays, we assessed the direct behavioral consequences of the spatial attentional bias we suggested had driven our electrophysiological findings. We conducted a dot-probe experiment to test the spatiotopic allocation of attention directly. If participants' attention was drawn back to the original location of test probes, then they ought to be faster to make arbitrary judgments about dot-probes appearing in that original location relative to a control location (Fukuda and Vogel, 2009). This was indeed the case. The judgment was significantly faster when the dot had appeared in the "attended" location relative to when it appeared in the "unattended" location 
$\left(F_{(1,12)}=11.87, p=0.005\right)$. This was unaffected by the dot-probe onset delay ( $p=0.22)$, and, more importantly, it was unaffected by whether the memory array had been supraliminal or subliminal ( $p=$ $0.55)$. There was a significant effect of matching versus mismatching location in both the supraliminal (493 vs $510 \mathrm{~ms}$, respectively, $\left.F_{(1,12)}=4.95, p=0.046\right)$ and the subliminal condition (505 vs $517 \mathrm{~ms}$, respectively, $\left.F_{(1,12)}=6.50, p=0.025\right)$. There were no significant differences in accuracy between match and mismatch trials in either the subliminal (95 vs $96 \%$, respectively, $p=0.579)$ or the supraliminal condition (95 vs 93\%, respectively, $p=0.307)$.

To validate our manipulation of liminality in this dot-probe experiment, as in the electrophysiological recording session, we immediately preceded and followed the experiment with the forced-choice explicitmemory search task. Before running the dot-probe experiment, participants' performance was not significantly better than chance in the "subliminal" condition $\left(d^{\prime}=\right.$ $\left.-0.16, t_{(12)}=-0.650, p=0.528\right)$ but was in the "supraliminal" condition $\left(d^{\prime}=1.75\right.$, $\left.t_{(12)}=4.744, p<0.001\right)$. After the dot-probe experiment, their performance was still not significantly better than chance when the arrays were presented for $63 \mathrm{~ms}\left(d^{\prime}=-0.07, t_{(12)}=-0.266, p=\right.$ $0.795)$ but was significantly better than chance when the arrays were presented for $243 \mathrm{~ms}\left(d^{\prime}=2.16, t_{(12)}=6.937, p<0.001\right)$.

\section{Discussion}

Recent functional imaging studies have demonstrated that retaining simple features of perceptual objects recruits early visual cortices; decoding the voxel-based activity within visual cortical areas V1, V2, and V3 can reveal accurately the particular items being intentionally stored in VSTM (Harrison and Tong, 2009; Serences et al., 2009). This would support the view that VSTM is mediated by domain-general control areas, such as posterior parietal and/or prefrontal cortex, interacting with brain regions involved in the original perception of the items (Passingham and Sakai, 2004). Consistent with this view, the contents of VSTM can be biased by the top-down allocation of attention, according to a particular task goal, as indexed by an N2pc (Astle et al., 2009; Kuo et al., 2009).

The current results demonstrate that the relationship between this posterior biasing mechanism and the contents of VSTM is bidirectional: not only do spatial biases influence the contents of memory, the contents of memory also drive spatial attentional biases when a match occurs with a currently perceived item. In both cases, spatial biases appear to modulate the current level of excitability in neural activity in visual areas, which participate in the coding of perceptual as well as memory arrays (Kuo et al., 2009). Surprisingly, even information that is task irrelevant and that does not have to be stored explicitly leaves a topographically organized trace that subsequently biases spatial attention. Moreover, the experiments presented here provide compelling evidence that objects stored automatically capture attention to their original location even when they are not consciously perceived (experiments 1 and 3). In addition to the electrophysiological evidence for memory-driven attentional capture elicited by objects in both supraliminal and subliminal masked arrays, we were able to measure the direct behavioral consequences of that capture using a dot-probe technique. This is especially surprising given that retaining the memory-array objects was in no way beneficial for performance, and the location of objects in memory did not predict the location of the upcoming dot; the capture was entirely automatic.

The dot-probe result also rules out one final alternative explanation for the electrophysiological result that the N2pc effect, rather than reflecting some spatially specific attentional process, actually indexes some form of object-based priming (Kristjánsson et al., 2001; Kuo et al., 2009). The task performed on the dot was completely unrelated to the perceptual match between the memory-array items and the test probe. That we observed a behavioral facilitation effect to a novel stimulus appearing in the same location cannot be imputed to simple perceptual priming and must instead result from the allocation of spatial attention. Furthermore, that the memory-based attentional capture effects survive pattern noise masking and occur over relatively lengthy intervals would also rule out visual priming as a likely explanation.

It has been demonstrated recently that incoming stimuli that participants fail to perceive can nonetheless capture attention (Ansorge et al., 2009). Here we show that previously presented stimuli that participants failed to perceive, and therefore unsurprisingly failed to recognize subsequently, can also capture spatial attention. In short, the subliminally presented stimuli leave a trace that retains the original spatial layout of the memory array and the particular configuration of lines that constitute each item. This trace is sufficient to drive spatial attention. However, the subliminal shapes are not assigned to higher-order categories, with the traces left by the subliminal memory arrays not affecting the symmetrical versus asymmetrical judgment on the test probe. An additional question, then, is what type of storage is responsi- 
ble for this effect? The traditional view of VSTM is of a capacitylimited, time-limited store. Because this store is topographically organized, it could be producing the effects that we observed. However, the traditional view of VSTM usually specifies that only items that participants intentionally retain are stored (Luria et al., 2010) and can capture attention (Olivers et al., 2006). It seems unlikely that our participants were intentionally storing the items; the memory array objects were essentially irrelevant for the task performed and, in some cases, were not consciously detectable. That said, it has been shown recently that large quantities of information may be retained in a fragile form of VSTM (Sligte et al., 2008) and that the existence of these fragile object representations can be evidenced using alternative means of probing "recognition," such as retrospective attentional cueing (Griffin and Nobre, 2003; Landman et al., 2003). The traces reported here are more robust than those stored in fragile VSTM (Sligte et al., 2008), because they survive combined forward and backward pattern masking. Nonetheless, like fragile VSTM, there seems to exist some form of item retention that cannot be evidenced by conventional recognition tests but that is nonetheless available. Our findings imply the existence of a previously undiscovered quality of VSTM or a parallel unconscious store which is also topographically organized. Either way, we demonstrate that objects that participants fail to perceive nonetheless leave a topographically organized trace and guide attention.

\section{References}

Ansorge U, Kiss M, Eimer M (2009) Goal-driven attentional capture by invisible colors: evidence from event-related potentials. Psychon Bull Rev 16:648-653.

Astle DE, Scerif G, Kuo BC, Nobre AC (2009) Spatial selection of features within perceived and remembered objects. Front Hum Neurosci 3:6.

Awh E, Jonides J (2001) Overlapping mechanisms of attention and spatial working memory. Trends Cogn Sci 5:119-126.

Cohen JY, Heitz RP, Schall JD, Woodman GF (2009) On the origin of eventrelated potentials indexing covert attentional selection during visual search. J Neurophysiol 102:2375-2386.

Cowan N (2001) The magical number 4 in short-term memory: a reconsideration of mental storage capacity. Behav Brain Sci 24:87-114; discussion $114-185$.

Dell'Acqua R, Sessa P, Toffanin P, Luria R, Jolicoeur P (2010) Orienting attention to objects in visual short-term memory. Neuropsychologia $48: 419-428$.
Eimer M, Kiss M (2010) An electrophysiological measure of access to representations in visual working memory. Psychophysiology 47:197-200.

Fabiani M, Ho J, Stinard A, Grattona G (2003) Multiple visual memory phenomena in a memory search task. Psychophysiology 40:472-485.

Fuggetta G, Pavone EF, Walsh V, Kiss M, Eimer M (2006) Cortico-cortical interactions in spatial attention: a combined ERP/TMS study. J Neurophysiol 95:3277-3280.

Fukuda K, Vogel EK (2009) Human variation in overriding attentional capture. J Neurosci 29:8726-8733.

Griffin IC, Nobre AC (2003) Orienting attention to locations in internal representations. J Cogn Neurosci 15:1176-1194.

Harrison SA, Tong F (2009) Decoding reveals the contents of visual working memory in early visual areas. Nature 458:632-635.

Hopf JM, Boelmans K, Schoenfeld MA, Luck SJ, Heinze HJ (2004) Attention to features precedes attention to locations in visual search: evidence from electromagnetic brain responses in humans. J Neurosci 24:18221832.

Kristjánsson A, Mackeben M, Nakayama K (2001) Rapid, object-based learning in the deployment of transient attention. Perception 30:13751387.

Kuo BC, Rao A, Lepsien J, Nobre AC (2009) Searching for targets within the spatial layout of visual short-term memory. J Neurosci 29:8032-8038.

Landman R, Spekreijse H, Lamme VA (2003) Large capacity storage of integrated objects before change blindness. Vision Res 43:149-164.

Luck SJ, Hillyard SA (1994) Spatial filtering during visual search: evidence from human electrophysiology. J Exp Psychol Hum Percept Perform 20:1000-1014.

Luria R, Sessa P, Gotler A, Jolicœur P, Dell'Acqua R (2010) Visual shortterm memory capacity for simple and complex objects. J Cogn Neurosci 22:496-512.

Olivers CN, Meijer F, Theeuwes J (2006) Feature-based memory-driven attentional capture: visual working memory content affects visual attention. J Exp Psychol Hum Percept Perform 32:1243-1265.

Passingham D, Sakai K (2004) The prefrontal cortex and working memory: physiology and brain imaging. Curr Opin Neurobiol 14:163-168.

Serences JT, Ester EF, Vogel EK, Awh E (2009) Stimulus-specific delay activity in human primary visual cortex. Psychol Sci 20:207-214.

Sligte IG, Scholte HS, Lamme VA (2008) Are there multiple visual shortterm memory stores? PLoS ONE 3:e1699.

Soto D, Hodsoll J, Rotshtein P, Humphreys GW (2008) Automatic guidance of attention from working memory. Trends Cogn Sci 12:342-348.

Sperling G (1960) The information available in brief visual presentations. Psychol Monogr 74:1-29.

Vogel EK, Machizawa MG (2004) Neural activity predicts individual differences in visual working memory capacity. Nature 428:748-751.

Woodman GF, Luck SJ (1999) Electrophysiological measurement of rapid shifts of attention during visual search. Nature 400:867-869. 